\title{
INTERPOLATION BETWEEN BREZIS-VÁZQUEZ AND POINCARÉ INEQUALITIES ON NONNEGATIVELY CURVED SPACES: SHARPNESS AND RIGIDITIES
}

\author{
ALEXANDRU KRISTÁLY AND ANIKÓ SZAKÁL
}

\begin{abstract}
This paper is devoted to investigate an interpolation inequality between the Brezis-Vázquez and Poincaré inequalities (shortly, BPV inequality) on nonnegatively curved spaces. As a model case, we first prove that the BPV inequality holds on any Minkowski space, by fully characterizing the existence and shape of its extremals. We then prove that if a complete Finsler manifold with nonnegative Ricci curvature supports the BPV inequality, then its flag curvature is identically zero. In particular, we deduce that a Berwald space of nonnegative Ricci curvature supports the BPV inequality if and only if it is isometric to a Minkowski space. Our arguments explore fine properties of Bessel functions, comparison principles, and anisotropic symmetrization on Minkowski spaces. As an application, we characterize the existence of nonzero solutions for a quasilinear PDE involving the Finsler-Laplace operator and a Hardy-type singularity on Minkowski spaces where the sharp BPV inequality plays a crucial role. The results are also new in the Riemannian/Euclidean setting.
\end{abstract}

\section{INTRODUCTION}

One of the most spectacular improvements of the classical unipolar Hardy inequality is due to Brezis and Vázquez [6] by establishing that for every bounded domain $\Omega \subset \mathbb{R}^{n}(n \geq 2)$ with $0 \in \Omega$ one has

$$
\int_{\Omega}|\nabla u(x)|^{2} \mathrm{~d} x \geq \frac{(n-2)^{2}}{4} \int_{\Omega} \frac{u(x)^{2}}{|x|^{2}} \mathrm{~d} x+j_{0}^{2}\left(\frac{\omega_{n}}{|\Omega|}\right)^{\frac{2}{n}} \int_{\Omega} u(x)^{2} \mathrm{~d} x, \quad \forall u \in W_{0}^{1,2}(\Omega),
$$

where $j_{0}=2.4048$ is the first positive zero of the Bessel function of first kind $J_{0}$, while $|\Omega|$ and $\omega_{n}$ denote the volumes of the set $\Omega$ and the $n$-dimensional Euclidean unit ball, respectively. In the limit case $n=2$, the inequality (BV) reduces precisely to the optimal Poincaré inequality.

The aforementioned inequalities constitute a continuous source of inspiration for further investigations not only in the Euclidean setting, see e.g. Adimurthi, Chaudhuri and Ramaswamy [1], Barbatis, Filippas and Tertikas [3], Ghoussoub and Moradifam [14, 15], but also on curved spaces. More precisely, such Sobolev-type inequalities behave quite naturally on Hadamard manifolds (simply connected, complete Riemannian/Finsler manifolds with nonpositive sectional/flag curvature), as shown e.g. by Carron [7, 8], Berchio, Ganguly and Grillo [5], D'Ambrosio and Dipierro [10], Kombe and Özaydin [16, 17], Farkas, Kristály and Varga [13], Kristály [18], Yang, Su and Kong [26]. This fact is not surprising since Hadamard manifolds are diffeomorphic to Euclidean spaces.

Our paper is devoted to study an inequality on nonnegatively curved spaces whose limit cases are the Brezis-Vázquez and (not necessarily the 2-dimensional) Poincaré inequalities.

In order to formulate the interpolation inequality, let $(M, F)$ be a complete $n$-dimensional reversible Finsler manifold ( $n \geq 2$ be an integer) and $\alpha \in\left[0, \frac{n-2}{2}\right]$ be fixed. If $\Omega \subset M$ is a bounded open set and $x_{0} \in \Omega$, we consider the Brezis-Poincaré-Vázquez inequality

$$
\int_{\Omega} F_{*}(x, D u(x))^{2} \mathrm{~d} V_{F}(x) \geq\left[\frac{(n-2)^{2}}{4}-\alpha^{2}\right] \int_{\Omega} \frac{u(x)^{2}}{d_{F}\left(x_{0}, x\right)^{2}} \mathrm{~d} V_{F}(x)+S_{\alpha}(\Omega) \int_{\Omega} u(x)^{2} \mathrm{~d} V_{F}(x), \forall u \in C_{0}^{\infty}(\Omega),
$$

(BPV)

2000 Mathematics Subject Classification. Primary: 53C23, 58J05; Secondary: 35R01, 35R06, 53C60, 33C10.

Key words and phrases. Brezis-Vázquez inequality; Poincaré inequality; Finsler manifold; Minkowski space; sharpness; extremals; Bessel functions. 
where

$$
S_{\alpha}(\Omega):=j_{\alpha}^{2}\left(\frac{\omega_{n}}{\operatorname{Vol}_{F}(\Omega)}\right)^{\frac{2}{n}},
$$

and $j_{\alpha}$ is the first positive zero of the Bessel function of the first kind $J_{\alpha}$. Hereafter, $F_{*}, d_{F}, \mathrm{~d} V_{F}$ and $\mathrm{Vol}_{F}$ denote the polar transform, the metric function, the canonical measure and Finslerian volume on $(M, F)$, respectively; for details, see Section 2.

In the classical Euclidean setting, (BPV) reduces to the Brezis-Vázquez inequality (BV) when $\alpha=0$, and to the optimal Poincaré inequality when $\alpha=\frac{n-2}{2}$.

In fact, our first main result shows that (BPV) holds on Minkowski spaces, the simplest Finslerian structures with vanishing flag curvature (i.e., $\mathbb{R}^{n}$ endowed with an arbitrary smooth norm). Without loss of generality, the Minkowski norm in $\left(\mathbb{R}^{n}, F\right)$ is scaled such that $B_{0}^{F}(1)=\left\{x \in \mathbb{R}^{n}: F(x)<1\right\}$ has volume $\omega_{n}$. As usual, a set $\Omega \subset \mathbb{R}^{n}$ has a Wulff shape if it is homothetic to $B_{0}^{F}(1)$. For further use, let $l_{F} \in(0,1]$ be the uniformity constant associated with $F$; we note that $l_{F}=1$ if and only if $F$ is Euclidean, see Section 2. By using anisotropic symmetrization arguments, see Alvino, Ferone, Lions and Trombetti [2] and Van Schaftingen [24], and fine convexity properties of the Hardy functional involving the uniformity constant $l_{F}$ on $\left(\mathbb{R}^{n}, F\right)$, we prove the following result.

Theorem 1.1. Let $\left(\mathbb{R}^{n}, F\right)$ be a Minkowski space, $n \geq 2$, and fix $\alpha \in\left[\frac{n-2}{2} \sqrt{1-l_{F}^{2}}, \frac{n-2}{2}\right]$. Then inequality (BPV) holds for every open bounded set $\Omega \subset \mathbb{R}^{n}$ and $x_{0} \in \Omega$.

Moreover, given an open set $\Omega \subset \mathbb{R}^{n}$, equality holds in (BPV) for some function belonging to the Sobolev space $W_{0}^{1,2}(\Omega)$ if and only if $\Omega$ has a Wulff shape and either $\alpha=0$ when $n=2$, or $\alpha>0$ when $n \geq 3$; in such cases, the extremal function has the form

$$
u^{\star}(x)=F(x)^{\frac{2-n}{2}} J_{\alpha}\left(\sqrt{S_{\alpha}(\Omega)} F(x)\right), x \in \Omega^{\star},
$$

where $\Omega^{\star}$ is the anisotropic symmetrization of $\Omega$.

Having the flat case (Theorem 1.1), a natural question arises: what about the (BPV) inequality on nonnegatively curved Finsler manifolds? The answer is given in the following rigidity result.

Theorem 1.2. Let $(M, F)$ be a complete $n$-dimensional reversible Finsler manifold $(n \geq 2)$ with nonnegative $n$-Ricci curvature, and $\alpha \in\left[0, \frac{n-2}{2}\right]$ be such that $\alpha>0$ whenever $n \geq 3$. If (BPV) holds for every open bounded set $\Omega \subset M$ and $x_{0} \in \Omega$, then the flag curvature of $(M, F)$ is identically zero.

The proof of Theorem 1.2 requires a fine analysis of Bessel functions combined with the BishopGromov volume comparison principle on Finsler manifolds.

Theorem 1.2 is new in the Riemannian setting as well; however, its conclusion in the particular case $\alpha=\frac{n-2}{2}$ can be obtained by the Rayleigh-Faber-Krahn inequality established by Cheng [9]. Indeed, when $(M, g)$ is an $n$-dimensional Riemannian manifold with nonnegative Ricci curvature endowed with its natural metric $d_{g}$ and canonical measure $\mathrm{d} V_{g}$, we have the Rayleigh-Faber-Krahn inequality

$$
\mu_{1}\left(B_{x}(\rho)\right):=\inf _{u \in W_{0}^{1,2}\left(B_{x}(\rho)\right) \backslash\{0\}} \frac{\int_{B_{x}(\rho)}|D u|(x)^{2} \mathrm{~d} V_{g}(x)}{\int_{B_{x}(\rho)} u(x)^{2} \mathrm{~d} V_{g}(x)} \leq \mu_{1}\left(B_{0}^{e}(\rho)\right)=\frac{j_{n / 2-1}^{2}}{\rho^{2}},
$$

where $B_{x}(\rho)=\left\{y \in M: d_{g}(x, y)<\rho\right\}$ and $B_{0}^{e}(\rho)$ is the $n$-dimensional Euclidean ball with center 0 and radius $\rho>0$; moreover, equality holds in (1.2) if and only if $B_{x}(\rho)$ is isometric to $B_{0}^{e}(\rho)$, see Cheng [9]. In this Riemannian setting, the validity of the inequality (BPV) with $\alpha=\frac{n-2}{2}$ (i.e., Poincaré inequality) implies equality in (1.2), thus the conclusion in Theorem 1.2 directly follows by Cheng's result. However, Cheng's approach - based on a careful analysis of Jacobi fields on normal coordinates of $(M, g)$ - cannot be adapted to our setting, where some singular terms also occur when $\alpha \neq \frac{n-2}{2}$. 
Theorems 1.1 and 1.2 can be elegantly summarized on Berwald spaces, by providing an analytic characterization of Minkowski spaces through the (BPV) inequality.

Theorem 1.3. Let $(M, F)$ be a complete $n$-dimensional reversible Berwald space $(n \geq 2)$ having nonnegative Ricci curvature, uniformity constant $l_{F} \in(0,1]$, and fix $\alpha \in\left[\frac{n-2}{2} \sqrt{1-l_{F}^{2}}, \frac{n-2}{2}\right]$ such that $\alpha>0$ whenever $n \geq 3$. Then the following two statements are equivalent:

(i) (BPV) holds for every open bounded set $\Omega \subset M$ and $x_{0} \in \Omega$;

(ii) $(M, F)$ is isometric to a Minkowski space.

As an application of the (BPV) inequality, we consider on a Minkowski space $\left(\mathbb{R}^{n}, F\right)$ the following quasilinear Dirichlet problem

$$
\left\{\begin{array}{l}
-\Delta_{F} u(x)-\left[\frac{(n-2)^{2}}{4}-\alpha^{2}\right] \frac{u(x)}{F(x)^{2}}+\lambda u(x)=|u(x)|^{p-2} u(x), \quad x \in B_{0}^{F}(1) ; \\
u \geq 0, u \in W_{0}^{1,2}\left(B_{0}^{F}(1)\right),
\end{array}\right.
$$

where $\Delta_{F} u=\operatorname{div}\left(J^{*}(D u(x))\right)$ is the Finsler-Laplace operator on $\left(\mathbb{R}^{n}, F\right), J^{*}$ being the Legendre transform associated to $F$, see Section 2. The following result characterizes the existence of nonzero solutions of problem $\left(\mathcal{P}_{\alpha, \lambda}\right)$ depending on the parameters $\alpha, \lambda \in \mathbb{R}$. As usual, $2^{*}$ denotes the critical Sobolev exponent $\left(2^{*}=2 n /(n-2)\right.$ if $n \geq 3$ and $2^{*}=\infty$ if $\left.n=2\right)$.

Theorem 1.4. Let $\left(\mathbb{R}^{n}, F\right)$ be a Minkowski space, $n \geq 2$, and fix $\alpha \in\left[\frac{n-2}{2} \sqrt{1-l_{F}^{2}}, \frac{n-2}{2}\right]$ such that $\alpha>0$ whenever $n \geq 3$. Let $p \in\left(2,2^{*}\right)$ be fixed. Then problem $\left(\mathcal{P}_{\alpha, \lambda}\right)$ has a nonzero solution if and only if $\lambda>-j_{\alpha}^{2}$.

Theorem 1.4 is known in the special case when $F$ is Euclidean and $\alpha=\frac{n-2}{2}$ (thus, the singular term disappears), see Willem [25]. The proof of Theorem 1.4 is variational, based on the mountain pass theorem and the validity of the sharp inequality (BPV) on Minkowski spaces.

The organization of the paper is the following. In Section 2 we recall basic notions from Finsler geometry (flag curvature, Ricci curvature, Bishop-Gromov volume comparison principle). In Section 3, before presenting the proof of Theorem 1.2, we recall some basic results from the theory of anisotropic symmetrization on Minkowski spaces. In Section 4 we prove Theorems 1.2 and 1.3; to complete this, we first establish some properties of Bessel functions which are interesting in their own right. Finally, in Section 5 we prove Theorem 1.4.

\section{Preliminaries on Finsler manifolds}

Let $M$ be a connected $n$-dimensional $C^{\infty}$-manifold and $T M=\bigcup_{x \in M} T_{x} M$ be its tangent bundle. The pair $(M, F)$ is called a reversible Finsler manifold if the continuous function $F: T M \rightarrow[0, \infty)$ satisfies the conditions:

(a) $F \in C^{\infty}(T M \backslash\{0\})$;

(b) $F(x, t v)=|t| F(x, v)$ for all $t \in \mathbb{R}$ and $(x, v) \in T M$;

(c) the $n \times n$ matrix

$$
g_{(x, v)}:=\left[g_{i j}(x, v)\right]_{i, j=1, \ldots, n}=\left[\frac{1}{2} \frac{\partial^{2}}{\partial v^{i} \partial v^{j}} F^{2}(x, v)\right]_{i, j=1, \ldots, n}, \quad \text { where } v=\sum_{i=1}^{n} v^{i} \frac{\partial}{\partial x^{i}},
$$

is positive definite for all $(x, v) \in T M \backslash\{0\}$. We will denote by $g_{v}$ the inner product on $T_{x} M$ induced by $(2.1)$.

If $g_{i j}(x)=g_{i j}(x, v)$ is independent of $v$ then $(M, F)=(M, g)$ is called a Riemannian manifold. A Minkowski space consists of a finite dimensional vector space $V$ (identified with $\mathbb{R}^{n}$ ) and a Minkowski norm which induces a Finsler metric on $V$ by translation, i.e., $F(x, v)$ is independent on the base point 
$x$; in such cases we often write $F(v)$ instead of $F(x, v)$. A Finsler manifold $(M, F)$ is called a locally Minkowski space if any point in $M$ admits a local coordinate system $\left(x^{i}\right)$ on its neighborhood such that $F(x, v)$ depends only on $v$ and not on $x$.

For every $(x, \alpha) \in T^{*} M$, the polar transform (or, co-metric) of $F$ is given by

$$
F_{*}(x, \alpha)=\sup _{v \in T_{x} M \backslash\{0\}} \frac{\alpha(v)}{F(x, v)} .
$$

Note that for every $x \in M$, the function $F_{*}(x, \cdot)$ is a Minkowski norm on $T_{x}^{*} M$.

The number

$$
l_{F}=\inf _{x \in M} l_{F}(x), \quad \text { where } \quad l_{F}(x):=\inf _{y, v, w \in T_{x} M \backslash\{0\}} \frac{g_{(x, v)}(y, y)}{g_{(x, w)}(y, y)},
$$

is the uniformity constant associated with $F$ which measures how far $(M, F)$ and $\left(M, F_{*}\right)$ are from Riemannian structures. In fact, one can see that $l_{F} \leq 1$, and $l_{F}=1$ if and only if $(M, F)$ is a Riemannian manifold. When $\left(\mathbb{R}^{n}, F\right)$ is a Minkowski space, we have that $l_{F} \in(0,1]$. The definition of $l_{F}$ in turn shows that

$$
\left[F_{*}(x, t \alpha+(1-t) \beta)\right]^{2} \leq t\left[F_{*}(x, \alpha)\right]^{2}+(1-t)\left[F_{*}(x, \beta)\right]^{2}-l_{F} t(1-t)\left[F_{*}(x, \beta-\alpha)\right]^{2}
$$

for all $x \in M, \alpha, \beta \in T_{x}^{*} M$ and $t \in[0,1]$.

Let $\pi^{*} T M$ be the pull-back bundle of the tangent bundle $T M$ generated by the natural projection $\pi: T M \backslash\{0\} \rightarrow M$, see Bao, Chern and Shen [4]. The vectors of the pull-back bundle $\pi^{*} T M$ are denoted by $(v ; w)$ with $(x, y)=v \in T M \backslash\{0\}$ and $w \in T_{x} M$. For simplicity, let $\left.\partial_{i}\right|_{v}=\left(v ; \partial /\left.\partial x^{i}\right|_{x}\right)$ be the natural local basis for $\pi^{*} T M$, where $v \in T_{x} M$. One can introduce on $\pi^{*} T M$ the fundamental tensor $g$ by $g_{(x, v)}:=g_{v}=g\left(\left.\partial_{i}\right|_{v},\left.\partial_{j}\right|_{v}\right)=g_{i j}(x, y)$, where $v=\left.y^{i}\left(\partial / \partial x^{i}\right)\right|_{x}$, see (2.1). Unlike the Levi-Civita connection in the Riemannian case, there is no unique natural connection in the Finsler geometry. Among these connections on the pull-back bundle $\pi^{*} T M$, we choose a torsion-free and almost metric-compatible linear connection on $\pi^{*} T M$, the so-called Chern connection. The coefficients of the Chern connection are denoted by $\Gamma_{j k}^{i}$, which are instead of the well-known Christoffel symbols from Riemannian geometry.

A Finsler manifold is of Berwald type if the coefficients $\Gamma_{i j}^{k}(x, y)$ in natural coordinates are independent of $y$. It is clear that Riemannian manifolds and (locally) Minkowski spaces are Berwald spaces. The Chern connection induces on $\pi^{*} T M$ the curvature tensor $R$. The Finsler manifold is complete if every geodesic segment $\sigma:[0, a] \rightarrow M$ can be extended to $\mathbb{R}$.

Let $u, v \in T_{x} M$ be two non-collinear vectors and $\mathcal{S}=\operatorname{span}\{u, v\} \subset T_{x} M$. By means of the curvature tensor $R$, the flag curvature associated with the flag $\{\mathcal{S}, v\}$ is

$$
\mathbf{K}(\mathcal{S} ; v)=\frac{g_{v}(R(U, V) V, U)}{g_{v}(V, V) g_{v}(U, U)-g_{v}^{2}(U, V)},
$$

where $U=(v ; u), V=(v ; v) \in \pi^{*} T M$. If $(M, F)$ is Riemannian, the flag curvature reduces to the sectional curvature which depends only on $\mathcal{S}$.

Take $v \in T_{x} M$ with $F(x, v)=1$ and let $\left\{e_{i}\right\}_{i=1}^{n}$ with $e_{n}=v$ be an orthonormal basis of $\left(T_{x} M, g_{v}\right)$ for $g_{v}$ from (2.1). Let $\mathcal{S}_{i}=\operatorname{span}\left\{e_{i}, v\right\}$ for $i=1, \ldots, n-1$. Then the Ricci curvature of $v$ is defined by $\operatorname{Ric}(v):=\sum_{i=1}^{n-1} \mathbf{K}\left(\mathcal{S}_{i} ; v\right)$.

Let $\mu$ be a positive smooth measure on $(M, F)$. Given $v \in T_{x} M \backslash\{0\}$, let $\sigma:(-\varepsilon, \varepsilon) \rightarrow M$ be the geodesic with $\dot{\sigma}(0)=v$ and decompose $\mu$ along $\sigma$ as $\mu=e^{-\psi} \operatorname{vol}_{\dot{\sigma}}$, where $\operatorname{vol}_{\dot{\sigma}}$ denotes the volume form of the Riemannian structure $g_{\dot{\sigma}}$. For $N \in[n, \infty]$, the $N$-Ricci curvature Ric $_{N}$ is defined by

$$
\operatorname{Ric}_{N}(v):=\operatorname{Ric}(v)+(\psi \circ \sigma)^{\prime \prime}(0)-\frac{(\psi \circ \sigma)^{\prime}(0)^{2}}{N-n},
$$

where the third term is understood as 0 if $N=\infty$ or if $N=n$ with $(\psi \circ \sigma)^{\prime}(0)=0$, and as $-\infty$ if $N=n$ with $(\psi \circ \sigma)^{\prime}(0) \neq 0$. 
Let $\sigma:[0, r] \rightarrow M$ be a piecewise smooth curve. The value $L_{F}(\sigma)=\int_{0}^{r} F(\sigma(t), \dot{\sigma}(t)) \mathrm{d} t$ denotes the integral length of $\sigma$. For $x_{1}, x_{2} \in M$, denote by $\Lambda\left(x_{1}, x_{2}\right)$ the set of all piecewise $C^{\infty}$ curves $\sigma:[0, r] \rightarrow M$ such that $\sigma(0)=x_{1}$ and $\sigma(r)=x_{2}$. Define the metric function $d_{F}: M \times M \rightarrow[0, \infty)$ by

$$
d_{F}\left(x_{1}, x_{2}\right)=\inf _{\sigma \in \Lambda\left(x_{1}, x_{2}\right)} L_{F}(\sigma) .
$$

The metric ball with center $x \in M$ and radius $\rho>0$ is defined by $B_{x}(\rho)=\left\{y \in M: d_{F}(x, y)<\rho\right\}$.

Let $\left\{\partial / \partial x^{i}\right\}_{i=1, \ldots, n}$ be a local basis for the tangent bundle $T M$, and $\left\{\mathrm{d} x^{i}\right\}_{i=1, \ldots, n}$ be its dual basis for $T^{*} M$. Consider $\tilde{B}_{x}(1)=\left\{y=\left(y^{i}\right): F\left(x, y^{i} \partial / \partial x^{i}\right)<1\right\} \subset \mathbb{R}^{n}$. The Busemann-Hausdorff volume form is defined by

$$
\mathrm{d} V_{F}(x)=\sigma_{F}(x) \mathrm{d} x^{1} \wedge \ldots \wedge \mathrm{d} x^{n},
$$

where $\sigma_{F}(x)=\frac{\omega_{n}}{\left|\tilde{B}_{x}(1)\right|}$. The Finslerian volume of an open set $S \subset M$ is $\operatorname{Vol}_{F}(S)=\int_{S} \mathrm{~d} V_{F}(x)$. When $\left(\mathbb{R}^{n}, F\right)$ is a Minkowski space, then $d_{F}\left(x_{1}, x_{2}\right)=F\left(x_{2}-x_{1}\right)$ and on account of $(2.6), \operatorname{Vol}_{F}\left(B_{x}(\rho)\right)=$ $\omega_{n} \rho^{n}$ for every $\rho>0$ and $x \in \mathbb{R}^{n}$.

On any Finsler manifold $(M, F)$ we have for every $x \in M$ that

$$
\lim _{\rho \rightarrow 0^{+}} \frac{\operatorname{Vol}_{F}\left(B_{x}(\rho)\right)}{\omega_{n} \rho^{n}}=1 .
$$

Let $(M, F)$ be a complete $n$-dimensional Finsler manifold with nonnegative $N$-Ricci curvature. Then the Bishop-Gromov volume comparison principle provides that the function

$$
\rho \mapsto \frac{\operatorname{Vol}_{F}\left(B_{x}(\rho)\right)}{\rho^{N}}, \quad \rho>0,
$$

is non-increasing for every $x \in M$. In particular, if $N=n$, then

$$
\operatorname{Vol}_{F}\left(B_{x}(\rho)\right) \leq \omega_{n} \rho^{n}, \forall x \in M, \rho>0 .
$$

Moreover, if equality holds in (2.8), then the flag curvature is identically zero, see Ohta [21], Shen [23].

The (distributional) derivative of $u: M \rightarrow \mathbb{R}$ at $x \in M$ is $D u(x)=\sum_{i=1}^{n} \frac{\partial}{\partial x^{i}} u(x) \mathrm{d} x^{i}$, and due to Ohta and Sturm [22], one has the eikonal equation

$$
F_{*}\left(x, D d_{F}\left(x_{0}, \cdot\right)(x)\right)=1 \text { for a.e. } x \in M \text {. }
$$

The Legendre transform $J^{*}: T^{*} M \rightarrow T M$ associates to each element $\xi \in T_{x}^{*} M$ the unique maximizer on $T_{x} M$ of the map $y \mapsto \xi(y)-\frac{1}{2} F(x, y)^{2}$. The gradient of $u$ is defined by $\nabla_{F} u(x)=J^{*}(x, D u(x))$. The Finsler-Laplace operator is given by

$$
\Delta_{F} u=\operatorname{div}\left(\nabla_{F} u\right)
$$

where $\operatorname{div}(X)=\frac{1}{\sigma_{F}} \frac{\partial}{\partial x^{i}}\left(\sigma_{F} X^{i}\right)$ for some vector field $X$ on $M$, and $\sigma_{F}$ comes from (2.6).

Consider the Sobolev space

$$
W^{1,2}(M, F):=\left\{u \in W_{\mathrm{loc}}^{1,2}(M): \int_{M} F_{*}(x, D u(x))^{2} \mathrm{~d} V_{F}(x)<+\infty\right\},
$$

associated with $(M, F)$ and let $W_{0}^{1,2}(M, F, \mathrm{~m})$ be the closure of $C_{0}^{\infty}(M)$ with respect to the norm

$$
\|u\|_{F}:=\left(\int_{M} F_{*}(x, D u(x))^{2} \mathrm{~d} V_{F}(x)+\int_{M} u(x)^{2} \mathrm{~d} V_{F}(x)\right)^{1 / 2} .
$$

When $\left(\mathbb{R}^{n}, F\right)$ is a Minkowski space, then $W_{0}^{1,2}(\Omega, F)$ is the usual Sobolev space $W_{0}^{1,2}(\Omega)$ for every open set $\Omega \subset \mathbb{R}^{n}$, see Kristály and Ohta [19]; indeed, in this case there exits $C_{0} \geq 1$ such that $C_{0}^{-1}|x| \leq F(x) \leq C_{0}|x|$ for every $x \in \mathbb{R}^{n}$. 


\section{Proof of Theorem 1.1}

Before to present the proof of Theorem 1.1, we recall some notions and results established in Alvino, Ferone, Lions and Trombetti [2] and Van Schaftingen [24] concerning anisotropic symmetrization.

Let $\left(\mathbb{R}^{n}, F\right)$ be a Minkowski space, $n \geq 2$. If $\Omega \subset \mathbb{R}^{n}$ is a measurable set, we denote by $\Omega^{\star}$ its anisotropic symmetrization defined as the open ball with center 0 such that $|\Omega|=\left|\Omega^{\star}\right|$. It is clear that $\Omega^{*}$ has a Wulff shape, homothetic to $B_{0}^{F}(1)=\left\{x \in \mathbb{R}^{n}: F(x)<1\right\}$. If $u: \mathbb{R}^{n} \rightarrow[0, \infty)$ is a function, then

$$
u^{\star}(x)=\sup \left\{c \in \mathbb{R}: x \in\{u>c\}^{\star}\right\}
$$

is the anisotropic (decreasing) symmetrization of $u$. Here, $\{u>c\}=\left\{x \in \mathbb{R}^{n}: u(x)>c\right\}$. The following results are valid:

- Anisotropic Cavalieri principle (see [24, Proposition 2.28]). Let $u: \mathbb{R}^{n} \rightarrow[0, \infty$ ) be a function vanishing at infinity with respect to $\cdot{ }^{\star}$. Then

$$
\int_{\mathbb{R}^{n}} u(x)^{2} \mathrm{~d} x=\int_{\mathbb{R}^{n}} u^{\star}(x)^{2} \mathrm{~d} x .
$$

- Anisotropic Pólya-Szegö inequality (see [2, Theorem 3.1] and [24, Theorem 6.8]). If $\Omega \subset \mathbb{R}^{n}$ is an open set and $u \in W_{0}^{1,2}(\Omega)_{+}=\left\{u \in W_{0}^{1,2}(\Omega): u \geq 0\right\}$, then $u^{\star} \in W_{0}^{1,2}\left(\Omega^{\star}\right)_{+}$and

$$
\int_{\Omega^{\star}} F_{*}\left(D u^{\star}(x)\right)^{2} \mathrm{~d} x \leq \int_{\Omega} F_{*}(D u(x))^{2} \mathrm{~d} x .
$$

- Anisotropic Hardy-Littlewood inequality (see [24, Proposition 2.28]). For every open $\Omega \subset \mathbb{R}^{n}$ and $u \in W_{0}^{1,2}(\Omega)_{+}$, one has

$$
\int_{\Omega} \frac{u(x)^{2}}{F(x)^{2}} \mathrm{~d} x \leq \int_{\Omega^{\star}} \frac{u^{\star}(x)^{2}}{F(x)^{2}} \mathrm{~d} x .
$$

Finally, we recall the anisotropic Hardy inequality from [24]: when $n \geq 3$, for every open set $\Omega \subset \mathbb{R}^{n}$ one has that

$$
\int_{\Omega} F_{*}(D u(x))^{2} \mathrm{~d} x \geq \frac{(n-2)^{2}}{4} \int_{\Omega} \frac{u(x)^{2}}{F(x)^{2}} \mathrm{~d} x, \quad \forall u \in W_{0}^{1,2}(\Omega)
$$

moreover, $\frac{(n-2)^{2}}{4}$ is optimal and never attained.

The following result is crucial in the study of extremal functions in the inequality (BPV).

Proposition 3.1. Let $\left(\mathbb{R}^{n}, F\right)$ be an n-dimensional reversible Minkowski space with the uniformity constant $l_{F}$ and fix $\mu \in\left[0, l_{F} \frac{(n-2)^{2}}{4}\right]$. Then for every open set $\Omega \subset \mathbb{R}^{n}$, the functional

$$
u \mapsto \mathcal{K}_{\mu}(u):=\int_{\Omega} F_{*}(D u(x))^{2} \mathrm{~d} x-\mu \int_{\Omega} \frac{u(x)^{2}}{F(x)^{2}} \mathrm{~d} x
$$

is positive and convex (thus, sequentially weakly lower semicontinous) on $W_{0}^{1,2}(\Omega)$. 
Proof. The positivity of $\mathcal{K}_{\mu}$ follows by the anisotropic Hardy inequality (3.1) and $0<l_{F} \leq 1$. Let us fix $0<t<1$ and $u, v \in W_{0}^{1,2}(\Omega)$. Then (2.3) and the anisotropic Hardy inequality (3.1) imply

$$
\begin{aligned}
\mathcal{K}_{\mu}(t u+(1-t) v)= & \int_{\Omega} F_{*}(x, t D u(x)+(1-t) D v(x))^{2} \mathrm{~d} x-\mu \int_{\Omega} \frac{(t u(x)+(1-t) v(x))^{2}}{F(x)^{2}} \mathrm{~d} x \\
\leq & t \int_{\Omega} F_{*}(x, D u(x))^{2} \mathrm{~d} x+(1-t) \int_{\Omega} F_{*}(x, D v(x))^{2} \mathrm{~d} x \\
& -l_{F} t(1-t) \int_{\Omega} F_{*}(x, D(v-u)(x))^{2} \mathrm{~d} x-\mu \int_{\Omega} \frac{(t u(x)+(1-t) v(x))^{2}}{F(x)^{2}} \mathrm{~d} x \\
= & t \mathcal{K}_{\mu}(u)+(1-t) \mathcal{K}_{\mu}(v) \\
& -t(1-t) l_{F} \int_{\Omega}\left(F_{*}(x, D(v-u)(x))^{2}-\mu l_{F}^{-1} \frac{(v(x)-u(x))^{2}}{F(x)^{2}}\right) \mathrm{d} x \\
\leq & t \mathcal{K}_{\mu}(u)+(1-t) \mathcal{K}_{\mu}(v),
\end{aligned}
$$

which concludes the proof.

Proof of Theorem 1.1. We split the proof into two parts.

Case I: $\alpha=0$ whenever $n=2$, or $\alpha>0$ whenever $n \geq 3$.

Let $\left(\mathbb{R}^{n}, F\right)$ be a Minkowski space, $\Omega \subset \mathbb{R}^{n}$ be an open bounded set and $\alpha \in\left[\frac{n-2}{2} \sqrt{1-l_{F}^{2}}, \frac{n-2}{2}\right]$ be fixed with the above properties; in particular, it turns out that $d_{F}\left(x_{0}, x\right)=F\left(x-x_{0}\right)$ for every $x_{0}, x \in \mathbb{R}^{n}$. After translation, we may also consider that $x_{0}=0 \in \Omega$. If

$$
\mu_{\alpha}(\Omega):=\inf _{u \in W_{0}^{1,2}(\Omega)}\left\{\int_{\Omega} F_{*}(D u(x))^{2} \mathrm{~d} x-\left[\frac{(n-2)^{2}}{4}-\alpha^{2}\right] \int_{\Omega} \frac{u(x)^{2}}{F(x)^{2}} \mathrm{~d} x: \int_{\Omega} u(x)^{2} \mathrm{~d} x=1\right\}
$$

it suffices to prove that

$$
\mu_{\alpha}(\Omega) \geq S_{\alpha}(\Omega)
$$

Moreover, since $F$ is absolutely homogeneous (so $F_{*}$ ), it is enough to consider only nonnegative test functions $u \in W_{0}^{1,2}(\Omega)_{+}$in $(3.2)$.

Let us consider a minimizing sequence for $\mu_{\alpha}(\Omega)$, i.e., $\left\{u_{k}\right\}_{k} \subset W_{0}^{1,2}(\Omega)_{+}$such that

$$
\int_{\Omega} u_{k}(x)^{2} \mathrm{~d} x=1, \quad \forall k \in \mathbb{N}
$$

and

$$
\int_{\Omega} F_{*}\left(D u_{k}(x)\right)^{2} \mathrm{~d} x-\left[\frac{(n-2)^{2}}{4}-\alpha^{2}\right] \int_{\Omega} \frac{u_{k}(x)^{2}}{F(x)^{2}} \mathrm{~d} x \rightarrow \mu_{\alpha}(\Omega) \text { as } k \rightarrow \infty .
$$

First, if $\alpha=0$ (when $n=2$ ), by relation (3.5) we have that $\left\{u_{k}\right\}_{k}$ is bounded in $W_{0}^{1,2}(\Omega)_{+}$. Second, if $\alpha>0$ (when $n \geq 3$ ), by the anisotropic Hardy inequality and (3.5), it follows again that $\left\{u_{k}\right\}_{k}$ is bounded in $W_{0}^{1,2}(\Omega)_{+}$. Accordingly, there exists $\tilde{u} \in W_{0}^{1,2}(\Omega)_{+}$such that (up to a subsequence) $\left\{u_{k}\right\}_{k}$ converges strongly to $\tilde{u}$ in $L^{2}(\Omega)$ and weakly to $\tilde{u}$ in $W_{0}^{1,2}(\Omega)$. By (3.4) we directly have

$$
\int_{\Omega} \tilde{u}(x)^{2} \mathrm{~d} x=1
$$

and by Proposition 3.1 and relation (3.5), 


$$
\begin{aligned}
\mu_{\alpha}(\Omega) & \leq \int_{\Omega} F_{*}(D \tilde{u}(x))^{2} \mathrm{~d} x-\left[\frac{(n-2)^{2}}{4}-\alpha^{2}\right] \int_{\Omega} \frac{\tilde{u}(x)^{2}}{F(x)^{2}} \mathrm{~d} x \\
& \leq \liminf _{k \rightarrow \infty}\left[\int_{\Omega} F_{*}\left(D u_{k}(x)\right)^{2} \mathrm{~d} x-\left[\frac{(n-2)^{2}}{4}-\alpha^{2}\right] \int_{\Omega} \frac{u_{k}(x)^{2}}{F(x)^{2}} \mathrm{~d} x\right] \\
& =\mu_{\alpha}(\Omega) .
\end{aligned}
$$

Thus, $\tilde{u} \in W_{0}^{1,2}(\Omega)_{+}$is a minimizer in (3.2). Let $u^{\star} \in W_{0}^{1,2}\left(\Omega^{\star}\right)_{+}$be the anisotropic symmetrization of $\tilde{u}$. By the anisotropic Pólya-Szegö, Hardy-Littlewood inequalities and Cavalieri principle one has

$$
\begin{aligned}
\mu_{\alpha}(\Omega) & =\int_{\Omega} F_{*}(D \tilde{u}(x))^{2} \mathrm{~d} x-\left[\frac{(n-2)^{2}}{4}-\alpha^{2}\right] \int_{\Omega} \frac{\tilde{u}(x)^{2}}{F(x)^{2}} \mathrm{~d} x \\
& \geq \int_{\Omega^{\star}} F_{*}\left(D u^{\star}(x)\right)^{2} \mathrm{~d} x-\left[\frac{(n-2)^{2}}{4}-\alpha^{2}\right] \int_{\Omega^{\star}} \frac{u^{\star}(x)^{2}}{F(x)^{2}} \mathrm{~d} x \\
& \geq \inf _{v \in W_{0}^{1,2}(\Omega)_{+}}\left\{\int_{\Omega^{\star}} F_{*}\left(D v^{\star}(x)\right)^{2} \mathrm{~d} x-\left[\frac{(n-2)^{2}}{4}-\alpha^{2}\right] \int_{\Omega^{\star}} \frac{v^{\star}(x)^{2}}{F(x)^{2}} \mathrm{~d} x: \int_{\Omega^{\star}}\left(v^{\star}\right)^{2} \mathrm{~d} x=1\right\}=: Q_{\alpha}(\Omega) .
\end{aligned}
$$

As above, one can prove that the latter infimum is attained; let $v^{\star} \in W_{0}^{1,2}\left(\Omega^{\star}\right)_{+}$be such a minimizer for $Q_{\alpha}(\Omega)$. We may assume that $v^{\star} \in C_{0}^{1}\left(\Omega^{\star}\right)_{+}$; otherwise, a density argument applies. Thus, there exists a non-increasing function $h:[0, \infty) \rightarrow[0, \infty)$ of class $C^{1}$ such that $v^{\star}(x)=h(\rho)$ where $\rho=F(x)$. Since $\Omega^{\star}$ has a Wulff shape, there exists $R_{\Omega}>0$ such that $\omega_{n} R_{\Omega}^{n}=\left|\Omega^{\star}\right|=|\Omega|$; moreover, $h\left(R_{\Omega}\right)=0$. From the absolute homogeneity of $F$ and relation (2.9), we have that

$$
F_{*}\left(D v^{\star}(x)\right)=F_{*}\left(h^{\prime}(\rho) D F(x)\right)=-h^{\prime}(\rho) F_{*}(D F(x))=-h^{\prime}(\rho) .
$$

Consequently, the function $h$ is a minimizer for

$$
Q_{\alpha}(\Omega)=\min _{w \in C^{1}\left(0, R_{\Omega}\right) ; w\left(R_{\Omega}\right)=0} \frac{\int_{0}^{R_{\Omega}}\left(w^{\prime}(\rho)\right)^{2} r^{n-1} \mathrm{~d} \rho-\left[\frac{(n-2)^{2}}{4}-\alpha^{2}\right] \int_{0}^{R_{\Omega}} w(\rho)^{2} \rho^{n-3} \mathrm{~d} \rho}{\int_{0}^{R_{\Omega}} w(\rho)^{2} \rho^{n-1} \mathrm{~d} \rho} .
$$

The corresponding Euler-Lagrange equation for $h$ reads as

$$
\left(h^{\prime}(\rho) \rho^{n-1}\right)^{\prime}+\left[\frac{(n-2)^{2}}{4}-\alpha^{2}\right] h(\rho) \rho^{n-3}+Q_{\alpha}(\Omega) h(\rho) \rho^{n-1}=0, \quad \rho \in\left(0, R_{\Omega}\right) .
$$

If $w(\rho):=\rho^{\frac{n-2}{2}} h(\rho),(3.7)$ reduces to the Bessel differential equation

$$
\rho^{2} w^{\prime \prime}(\rho)+\rho w^{\prime}(\rho)+\left(Q_{\alpha}(\Omega) \rho^{2}-\alpha^{2}\right) w(\rho)=0 .
$$

Accordingly, (3.7) has the general solution

$$
h(\rho)=c_{0} \rho^{\frac{2-n}{2}} J_{\alpha}\left(\sqrt{Q_{\alpha}(\Omega)} \rho\right)+c_{1} \rho^{\frac{2-n}{2}} Y_{\alpha}\left(\sqrt{Q_{\alpha}(\Omega)} \rho\right), \quad \rho \in\left(0, R_{\Omega}\right),
$$

where $c_{0}, c_{1} \in \mathbb{R}$, while $J_{\alpha}$ and $Y_{\alpha}$ are the Bessel functions of the first and second kind, respectively. Since $Y_{\alpha}$ is singular at the origin, we choose $c_{1}=0$; otherwise, $v^{\star}(x)=h(F(x))$ will not belong to $W_{0}^{1,2}\left(\Omega^{\star}\right)$. Furthermore, since $h\left(R_{\Omega}\right)=0$, it turns out that $\sqrt{Q_{\alpha}(\Omega)} R_{\Omega}=j_{\alpha}$, where $j_{\alpha}$ is the first positive zero of $J_{\alpha}$, which gives that

$$
Q_{\alpha}(\Omega)=S_{\alpha}(\Omega)=j_{\alpha}^{2}\left(\frac{\omega_{n}}{|\Omega|}\right)^{\frac{2}{n}},
$$

which implies (3.3), i.e, the validity of $(\mathbf{B P V})$ on $\left(\mathbb{R}^{n}, F\right)$. 
If equality holds in (BPV), then we have that $\mu_{\alpha}(\Omega)=S_{\alpha}(\Omega)$. The latter relation implies that in (3.6) we have equality; in particular, we have equality in the the Pólya-Szegö inequality, i.e.,

$$
\int_{\Omega} F_{*}(D \tilde{u}(x))^{2} \mathrm{~d} x=\int_{\Omega^{\star}} F_{*}\left(D u^{\star}(x)\right)^{2} \mathrm{~d} x .
$$

Due to Esposito and Trombetti [12, Theorem 5.1], the latter relation implies that $\Omega=\Omega^{*}$ (up to translations) and $\tilde{u}$ agrees almost everywhere (up to constant multiplication) with

$$
u^{\star}(x)=v^{\star}(x)=F(x)^{\frac{2-n}{2}} J_{\alpha}\left(\sqrt{S_{\alpha}(\Omega)} F(x)\right), x \in \Omega^{*},
$$

see (3.8). The asymptotic properties

$$
J_{\alpha}(t) \sim \frac{1}{\Gamma(\alpha+1)}\left(\frac{t}{2}\right)^{\alpha} \text { and } J_{\alpha}^{\prime}(t) \sim \frac{1}{2 \Gamma(\alpha)}\left(\frac{t}{2}\right)^{\alpha-1} \text { for } t \ll 1,
$$

immediately imply that $u^{\star} \in W_{0}^{1,2}\left(\Omega^{\star}\right)$.

Conversely, when $\Omega=\Omega^{\star}$, one clearly has $\mu_{\alpha}(\Omega)=S_{\alpha}(\Omega)$, and $u^{\star}$ is an extremal function in (BPV).

Case II: $\alpha=0$ whenever $n \geq 3$. In this case, we necessarily have that $l_{F}=1$, i.e., $F$ is Euclidean, thus we may proceed as in Brezis and Vázquez [6]. Applying again (anisotropic) symmetrization, it is enough to prove (BPV) only for symmetrized functions $v \in W_{0}^{1,2}\left(\Omega^{\star}\right)$. Let $v(x)=h(\rho)$ with $\rho=F(x)$, $x \in \Omega=\Omega^{\star}$. Note that $h\left(R_{\Omega}\right)=0$, where $R_{\Omega}=\left(\frac{|\Omega|}{\omega_{n}}\right)^{\frac{1}{n}}$. Let $w(\rho):=\rho^{\frac{n-2}{2}} h(\rho)$ with $\rho=F(x)$. Since $n \geq 3$, it turns out that $w(0)=0$. Moreover, since $w\left(R_{\Omega}\right)=0$, an integration by parts gives

$$
\int_{\Omega^{\star}} F_{*}(D v(x))^{2} \mathrm{~d} x=n \omega_{n} \int_{0}^{R_{\Omega}} \rho\left(w^{\prime}(\rho)\right)^{2} \mathrm{~d} \rho+n \omega_{n} \frac{(n-2)^{2}}{4} \int_{0}^{R_{\Omega}} \rho^{-1} w(\rho)^{2} \mathrm{~d} \rho .
$$

Furthermore, one has

$$
\int_{\Omega^{\star}} \frac{v(x)^{2}}{F(x)^{2}} \mathrm{~d} x=n \omega_{n} \int_{0}^{R_{\Omega}} \rho^{-1} w(\rho)^{2} \mathrm{~d} \rho
$$

and

$$
\int_{\Omega^{\star}} v(x)^{2} \mathrm{~d} x=n \omega_{n} \int_{0}^{R_{\Omega}} \rho w(\rho)^{2} \mathrm{~d} \rho .
$$

The above calculations show that in order to prove (BPV), it remains to check

$$
\int_{0}^{R_{\Omega}} \rho\left(w^{\prime}(\rho)\right)^{2} \mathrm{~d} \rho \geq S_{0}(\Omega) \int_{0}^{R_{\Omega}} \rho w(\rho)^{2} \mathrm{~d} \rho,
$$

which is nothing but the optimal 2-dimensional Poincaré inequality.

The equality in (BPV) would imply equality in (3.10). This would imply, similarly to Case I that $\Omega=\Omega^{\star}$ and $w(\rho)=J_{0}\left(\sqrt{S_{0}(\Omega)} \rho\right)$. But $v(x)=F(x)^{\frac{2-n}{2}} J_{0}\left(\sqrt{S_{0}(\Omega)} F(x)\right)$ does not belong to $W_{0}^{1,2}\left(\Omega^{\star}\right)$, see $(3.9)$.

Remark 3.1. A similar inequality to (BPV) can be stated also on not necessarily reversible Minkowski spaces. In such a setting, anisotropic symmetrization should be applied for positively homogeneous Minkowski norms, where a set having a Wulff shape is homothetic to the backward metric balls, see Van Schaftingen [24]. 


\section{Proof of Theorems 1.2 And 1.3}

In order to provide the proof of Theorems 1.2 and 1.3, we need some auxiliary results.

Proposition 4.1. Let $r>0$ and $f:(0, r] \rightarrow \mathbb{R}$ be a non-increasing function such that $f(r)=0$ and $(M, F)$ be a complete $n$-dimensional reversible Finsler manifold $(n \geq 2)$ with nonnegative $n$-Ricci curvature. Then for every fixed $x_{0} \in M$, one has

$$
\int_{B_{x_{0}}(r)} f\left(d_{F}\left(x_{0}, x\right)\right) \mathrm{d} V_{F}(x)=\int_{0}^{r} \mathrm{~A}_{x_{0}}(\rho) f(\rho) \mathrm{d} \rho,
$$

where $\mathrm{A}_{x_{0}}(\rho):=\frac{\mathrm{d}}{\mathrm{d} \rho} \operatorname{Vol}_{F}\left(B_{x_{0}}(\rho)\right)=\lim \sup _{\delta \rightarrow 0} \frac{\operatorname{Vol}_{F}\left(B_{x_{0}}(\rho+\delta)\right)-\operatorname{Vol}_{F}\left(B_{x_{0}}(\rho)\right)}{\delta}$ denotes the area of the sphere $\partial B_{x_{0}}(\rho)=\left\{y \in M: d_{F}\left(x_{0}, y\right)=\rho\right\}$.

Proof. By the Bishop-Gromov volume comparison principle on $(M, F)$ we have that $\rho \mapsto \frac{\operatorname{Vol}_{F}\left(B_{x_{0}}(\rho)\right)}{\rho^{n}}$ is non-increasing on $(0, \infty)$; in particular, $\rho \mapsto \operatorname{Vol}_{F}\left(B_{x_{0}}(\rho)\right)$ is differentiable a.e. on $[0, \infty)$. Let $l_{0}=\lim _{\rho \rightarrow 0} f(\rho)$. By using the layer cake representation together with the facts that $f:(0, r] \rightarrow \mathbb{R}$ is non-increasing and $f(r)=0$, an integration by parts gives

$$
\begin{aligned}
\int_{B_{x_{0}}(r)} f\left(d_{F}\left(x_{0}, x\right)\right) \mathrm{d} V_{F}(x) & =\int_{0}^{l_{0}} \operatorname{Vol}_{F}\left(\left\{x \in B_{x_{0}}(r): f\left(d_{F}\left(x_{0}, x\right)\right)>t\right\}\right) \mathrm{d} t \\
& \left.=\int_{r}^{0} \operatorname{Vol}_{F}\left(B_{x_{0}}(\rho)\right) f^{\prime}(\rho) \mathrm{d} \rho \quad \text { [change of variables } t=f(\rho)\right] \\
& =-\int_{0}^{r} \operatorname{Vol}_{F}\left(B_{x_{0}}(\rho)\right) f^{\prime}(\rho) \mathrm{d} \rho \\
& =\int_{0}^{r} \frac{\mathrm{d}}{\mathrm{d} \rho} \operatorname{Vol}_{F}\left(B_{x_{0}}(\rho)\right) f(\rho) \mathrm{d} \rho,
\end{aligned}
$$

which concludes the proof.

In the sequel we need some fine properties of Bessel functions of the first kind; we first recall some basic properties of them which are well known in the literature, see e.g. Erdélyi, Magnus, Oberhettinger and Tricomi [11]. The Bessel function of the first kind with order $\alpha \in \mathbb{R}$ is the solution of the differential equation

$$
t^{2} y^{\prime \prime}(t)+t y^{\prime}(t)+\left(t^{2}-\alpha^{2}\right)=0
$$

which is nonsingular at the origin; we denote it by $J_{\alpha}$. The function $J_{\alpha}$ has the following recurrence relations

$$
\begin{gathered}
J_{\alpha+1}(t)+J_{\alpha-1}(t)=\frac{2 \alpha}{t} J_{\alpha}(t), t>0 ; \\
J_{\alpha}^{\prime}(t)=-J_{\alpha+1}(t)+\frac{\alpha}{t} J_{\alpha}(t), t>0 ; \\
J_{\alpha+1}^{\prime}(t)=J_{\alpha}(t)-\frac{\alpha+1}{t} J_{\alpha+1}(t), t>0,
\end{gathered}
$$

where $J_{\alpha}^{\prime}$ is the derivative of $J_{\alpha}$. We know that the positive zeros of $J_{\alpha}$ form an increasing sequence $\left\{j_{\alpha, k}\right\}_{k \in \mathbb{N}}$, and the Mittag-Leffler expansion yields for every $\alpha>0$ that

$$
\begin{gathered}
\frac{J_{\alpha+1}(t)}{J_{\alpha}(t)}=\sum_{k \geq 1} \frac{2 t}{j_{\alpha, k}^{2}-t^{2}}, \quad|t|<j_{\alpha, 1} ; \\
t \frac{J_{\alpha}^{\prime}(t)}{J_{\alpha}(t)}=\alpha-\sum_{k \geq 1} \frac{2 t^{2}}{j_{\alpha, k}^{2}-t^{2}}, \quad|t|<j_{\alpha, 1} .
\end{gathered}
$$


In particular, by (4.5) we easily obtain for $\alpha>-1$ the Rayleigh sum

$$
\sum_{k \geq 1} \frac{1}{j_{\alpha, k}^{2}}=\frac{1}{4(\alpha+1)} .
$$

For simplicity, we use the notation $j_{\alpha}:=j_{\alpha, 1}$.

Proposition 4.2. Let $n \geq 2$ be an integer and $\alpha \in\left[0, \frac{n-2}{2}\right]$. Then the following properties hold:

(i) for every $\beta \in[0,2]$, the function $h_{1}(t):=t^{\beta-n} J_{\alpha}^{2}\left(j_{\alpha} t\right)$ is non-increasing on $(0,1]$;

(ii) the function $h_{2}(t):=t^{1-n} J_{\alpha}\left(j_{\alpha} t\right) J_{\alpha+1}\left(j_{\alpha} t\right)$ is non-increasing on $(0,1]$;

(iii) the function $h_{3}(t):=t^{2-n} J_{\alpha+1}\left(j_{\alpha} t\right)\left[J_{\alpha+1}\left(j_{\alpha} t\right)-\frac{n+2 \alpha}{j_{\alpha} t} J_{\alpha}\left(j_{\alpha} t\right)\right]$ is non-decreasing on $(0,1]$.

Proof. (i) It is enough to prove that $t \mapsto \tilde{h}_{1}(t):=\sqrt{h_{1}(t)}=t^{\frac{\beta-n}{2}} J_{\alpha}\left(j_{\alpha} t\right)$ is non-increasing on $(0,1)$. Since $J_{\alpha}\left(j_{\alpha} t\right) \neq 0$ for $t \in(0,1)$, by relation (4.6) we have

$$
\tilde{h}_{1}^{\prime}(t) \frac{t^{\frac{2+n-\beta}{2}}}{J_{\alpha}\left(j_{\alpha} t\right)}=\frac{\beta-n}{2}+j_{\alpha} t \frac{J_{\alpha}^{\prime}\left(j_{\alpha} t\right)}{J_{\alpha}\left(j_{\alpha} t\right)}=\frac{\beta-n}{2}+\alpha-\sum_{k \geq 1} \frac{2\left(j_{\alpha} t\right)^{2}}{j_{\alpha, k}^{2}-\left(j_{\alpha} t\right)^{2}} \leq \frac{\beta-n}{2}+\alpha \leq 0 .
$$

(ii) By using relations (4.3), (4.4), (4.5) and (4.7), we obtain for every $t \in(0,1)$ that

$$
\begin{aligned}
h_{2}^{\prime}(t) \frac{t^{n}}{J_{\alpha}^{2}\left(j_{\alpha} t\right)} & =-j_{\alpha} t \frac{J_{\alpha+1}^{2}\left(j_{\alpha} t\right)}{J_{\alpha}^{2}\left(j_{\alpha} t\right)}-n \frac{J_{\alpha+1}\left(j_{\alpha} t\right)}{J_{\alpha}\left(j_{\alpha} t\right)}+j_{\alpha} t \\
& \leq-n \frac{J_{\alpha+1}\left(j_{\alpha} t\right)}{J_{\alpha}\left(j_{\alpha} t\right)}+j_{\alpha} t=j_{\alpha} t\left[-2 n \sum_{k \geq 1} \frac{1}{j_{\alpha, k}^{2}-\left(j_{\alpha} t\right)^{2}}+1\right] \\
& \leq j_{\alpha} t\left[-2 n \sum_{k \geq 1} \frac{1}{j_{\alpha, k}^{2}}+1\right]=j_{\alpha} t\left[-\frac{n}{2(\alpha+1)}+1\right] \leq 0 .
\end{aligned}
$$

(iii) A similar reasoning as in (ii) gives for every $t \in(0,1)$ that

$$
\begin{aligned}
h_{3}^{\prime}(t) \frac{j_{\alpha} t^{n}}{J_{\alpha}^{2}\left(j_{\alpha} t\right)\left[2\left(j_{\alpha} t\right)^{2}+n(n+2 \alpha)\right]} & =\frac{J_{\alpha+1}\left(j_{\alpha} t\right)}{J_{\alpha}\left(j_{\alpha} t\right)}-\frac{(n+2 \alpha) j_{\alpha} t}{2\left(j_{\alpha} t\right)^{2}+n(n+2 \alpha)} \\
& =j_{\alpha} t\left[2 \sum_{k \geq 1} \frac{1}{j_{\alpha, k}^{2}-\left(j_{\alpha} t\right)^{2}}-\frac{(n+2 \alpha)}{2\left(j_{\alpha} t\right)^{2}+n(n+2 \alpha)}\right] \\
& \geq j_{\alpha} t\left[\frac{1}{2(\alpha+1)}-\frac{1}{n}\right] \geq 0,
\end{aligned}
$$

which concludes the proof.

The following auxiliary result provides an unusual rigidity in the theory of functional inequalities involving Bessel functions.

Proposition 4.3. Let $r>0$ be a real number, $n \geq 2$ be an integer, and $\alpha \in\left[0, \frac{n-2}{2}\right]$ be such $\alpha>0$ whenever $n \geq 3$. Assume that a function $f:(0, r] \rightarrow[0, \infty)$ satisfies the following properties:

(a) $\liminf \operatorname{in}_{t \rightarrow 0} \frac{f(t)}{t^{n}}=1$;

(b) the function $t \mapsto \frac{f(t)}{t^{n}}$ is non-increasing on $(0, r)$;

(c) $\int_{0}^{1} f(r t) t^{-n+1}\left[J_{\alpha+1}^{2}\left(j_{\alpha} t\right)-2\left(\frac{n-2}{2}-\alpha\right) \frac{J_{\alpha}^{\prime}\left(j_{\alpha} t\right) J_{\alpha}\left(j_{\alpha} t\right)}{j_{\alpha} t}-J_{\alpha}^{2}\left(j_{\alpha} t\right)\right] \mathrm{d} t \geq 0$.

Then $f(t)=t^{n}$ for every $t \in(0, r)$. 
Proof. Two cases are distinguished, depending on $\alpha$ and $n$.

Case I: $\alpha>0$ whenever $n \geq 3$. For simplicity of notation, let us introduce the function $H_{\alpha}:(0,1] \rightarrow$ $\mathbb{R}$ defined by

$$
H_{\alpha}(t):=J_{\alpha+1}^{2}\left(j_{\alpha} t\right)-2\left(\frac{n-2}{2}-\alpha\right) \frac{J_{\alpha}^{\prime}\left(j_{\alpha} t\right) J_{\alpha}\left(j_{\alpha} t\right)}{j_{\alpha} t}-J_{\alpha}^{2}\left(j_{\alpha} t\right) .
$$

By the integral identities

$$
\int_{0}^{1} t J_{\alpha+1}^{2}\left(j_{\alpha} t\right) \mathrm{d} t=\frac{J_{\alpha+1}^{2}\left(j_{\alpha}\right)}{2}, \quad \int_{0}^{1} J_{\alpha}\left(j_{\alpha} t\right) J_{\alpha}^{\prime}\left(j_{\alpha} t\right) \mathrm{d} t=0, \quad \int_{0}^{1} t J_{\alpha}^{2}\left(j_{\alpha} t\right) \mathrm{d} t=-\frac{J_{\alpha-1}\left(j_{\alpha}\right) J_{\alpha+1}\left(j_{\alpha}\right)}{2},
$$

see formula (10.22.5) from [20] and relation (4.2), we have that

$$
\int_{0}^{1} t H_{\alpha}(t) \mathrm{d} t=0
$$

According to relation (4.5), the function $t \mapsto \frac{J_{\alpha+1}^{2}\left(j_{\alpha} t\right)}{J_{\alpha}^{2}\left(j_{\alpha} t\right)}$ is increasing on $(0,1)$ and one has

$$
\lim _{t \rightarrow 0} \frac{J_{\alpha+1}^{2}\left(j_{\alpha} t\right)}{J_{\alpha}^{2}\left(j_{\alpha} t\right)}=0 \text { and } \lim _{t \rightarrow 1} \frac{J_{\alpha+1}^{2}\left(j_{\alpha} t\right)}{J_{\alpha}^{2}\left(j_{\alpha} t\right)}=+\infty .
$$

In a similar way, by (4.6), the function $t \mapsto \frac{J_{\alpha}^{\prime}\left(j_{\alpha} t\right)}{j_{\alpha} t J_{\alpha}\left(j_{\alpha} t\right)}$ is decreasing on $(0,1)$ and we have

$$
\lim _{t \rightarrow 0} \frac{J_{\alpha}^{\prime}\left(j_{\alpha} t\right)}{j_{\alpha} t J_{\alpha}\left(j_{\alpha} t\right)}=+\infty \text { and } \lim _{t \rightarrow 1} \frac{J_{\alpha}^{\prime}\left(j_{\alpha} t\right)}{j_{\alpha} t J_{\alpha}\left(j_{\alpha} t\right)}=-\infty
$$

The latter properties imply that the equation $H_{\alpha}(t)=0$ has a unique solution on $(0,1)$; let us denote by $t_{\alpha}^{0} \in(0,1)$ this element. The above analysis also shows that

$$
H_{\alpha}(t)<0, \forall t \in\left(0, t_{\alpha}^{0}\right) \text { and } H_{\alpha}(t)>0, \forall t \in\left(t_{\alpha}^{0}, 1\right] .
$$

Due to (a) and (b), it follows that

$$
f(t) \leq t^{n}, \quad \forall t \in(0, r)
$$

Relation (4.10) and the asymptotic properties (3.9) of the Bessel function imply that the terms within inequality (c) are well defined. Let $g:(0, r] \rightarrow[0,1]$ be defined by

$$
g(t)=1-\frac{f(t)}{t^{n}}
$$

By (a) and (b), it follows that $\limsup _{t \rightarrow 0} g(t)=0$ and $g$ is non-decreasing on $(0, r)$. Since $f(t)=$ $t^{n}-g(t) t^{n}$, by means of (4.8), the inequality in (c) can be transformed equivalently into

$$
\int_{0}^{1} g(r t) t H_{\alpha}(t) \mathrm{d} t \leq 0
$$

We are going to prove that $g \equiv 0$ on $(0, r)$. To see this, we have

$$
\begin{aligned}
0 & \left.\geq \int_{0}^{1} g(r t) t H_{\alpha}(t) \mathrm{d} t=\int_{0}^{t_{\alpha}^{0}} g(r t) t H_{\alpha}(t) \mathrm{d} t+\int_{t_{\alpha}^{0}}^{1} g(r t) t H_{\alpha}(t) \mathrm{d} t \quad \text { [see }(4.11)\right] \\
& \left.\geq g\left(r t_{\alpha}^{0}\right) \int_{0}^{t_{\alpha}^{0}} t H_{\alpha}(t) \mathrm{d} t+\int_{t_{\alpha}^{0}}^{1} g(r t) t H_{\alpha}(t) \mathrm{d} t \quad \text { [see (4.9) and monotonicity of } g\right] \\
& =-g\left(r t_{\alpha}^{0}\right) \int_{t_{\alpha}^{0}}^{1} t H_{\alpha}(t) \mathrm{d} t+\int_{t_{\alpha}^{0}}^{1} g(r t) t H_{\alpha}(t) \mathrm{d} t \quad[\text { see }(4.8)] \\
& =\int_{t_{\alpha}^{0}}^{1}\left[-g\left(r t_{\alpha}^{0}\right)+g(r t)\right] t H_{\alpha}(t) \mathrm{d} t .
\end{aligned}
$$


By (4.9) and the monotonicity of $g$ again, we necessarily have that $g(r t)=g\left(r t_{\alpha}^{0}\right)$ for every $t \in\left(t_{\alpha}^{0}, 1\right)$. Having this relation in mind, we have similarly as above that

$$
\begin{aligned}
0 & \geq \int_{0}^{1} g(r t) t H_{\alpha}(t) \mathrm{d} t=\int_{0}^{t_{\alpha}^{0}} g(r t) t H_{\alpha}(t) \mathrm{d} t+g\left(r t_{\alpha}^{0}\right) \int_{t_{\alpha}^{0}}^{1} t H_{\alpha}(t) \mathrm{d} t \\
& =\int_{0}^{t_{\alpha}^{0}} g(r t) t H_{\alpha}(t) \mathrm{d} t-g\left(r t_{\alpha}^{0}\right) \int_{0}^{t_{\alpha}^{0}} t H_{\alpha}(t) \mathrm{d} t \\
& =\int_{0}^{t_{\alpha}^{0}}\left[g(r t)-g\left(r t_{\alpha}^{0}\right)\right] t H_{\alpha}(t) \mathrm{d} t .
\end{aligned}
$$

Again, by (4.9) and the monotonicity of $g$ we have $g(r t)=g\left(r t_{\alpha}^{0}\right)$ for every $t \in\left(0, t_{\alpha}^{0}\right)$. Accordingly, $g(r t)=g\left(r t_{\alpha}^{0}\right)$ for every $t \in(0,1)$. Since $\lim _{\sup _{t \rightarrow 0}} g(t)=0$, we have that $g \equiv 0$ on $(0,1)$, which concludes the proof in Case I.

Case II: $\alpha=0$ whenever $n=2$. The proof is analogous to Case I; the only difference is that instead

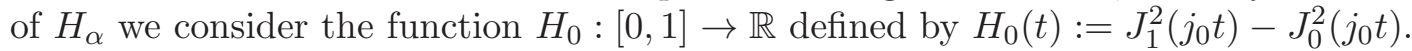

We are now ready to prove Theorem 1.2.

Proof of Theorem 1.2. We distinguish two cases.

Case I: $\alpha>0$ whenever $n \geq 3$. Let us fix $r>0$ arbitrarily. We are going to prove that the function

$$
u(x):=d_{F}\left(x_{0}, x\right)^{\frac{2-n}{2}} J_{\alpha}\left(j_{\alpha} \frac{d_{F}\left(x_{0}, x\right)}{r}\right), x \in B_{x_{0}}(r),
$$

can be used as a test function in (BPV) on the open set $B_{x_{0}}(r)$. To do this, we construct a sequence of functions with suitable convergence properties. More precisely, for every $k \in \mathbb{N}$ with $k>\frac{1}{r}+1$, we consider the Lipschitz function $u_{k}: B_{x_{0}}(r) \rightarrow \mathbb{R}$ defined by

$$
u_{k}(x)=\left(\max \left\{\frac{1}{k}, d_{F}\left(x_{0}, x\right)\right\}\right)^{\frac{2-n}{2}} J_{\alpha}\left(j_{\alpha} \frac{\min \left\{\left(1+\frac{1}{k}\right) d_{F}\left(x_{0}, x\right), r\right\}}{r}\right) .
$$

Since $(M, F)$ is complete, the set $\operatorname{supp}\left(u_{k}\right)=\overline{B_{x_{0}}\left(\frac{r k}{k+1}\right)}$ is compact. Therefore, by density reasons, $u_{k}$ can be uses as test functions in (BPV) on $B_{x_{0}}(r)$, i.e., for every $k \in \mathbb{N}$ one has

$$
\begin{aligned}
\int_{B_{x_{0}}(r)} F_{*}\left(x, D u_{k}(x)\right)^{2} \mathrm{~d} V_{F}(x) \geq & {\left[\frac{(n-2)^{2}}{4}-\alpha^{2}\right] \int_{B_{x_{0}}(r)} \frac{u_{k}(x)^{2}}{d_{F}\left(x_{0}, x\right)^{2}} \mathrm{~d} V_{F}(x) } \\
& +S_{\alpha}\left(B_{x_{0}}(r)\right) \int_{B_{x_{0}}(r)} u_{k}(x)^{2} \mathrm{~d} V_{F}(x) .
\end{aligned}
$$

Moreover, it immediately yields that

$$
\lim _{k \rightarrow \infty} u_{k}(x)=u(x) \text { and } \lim _{k \rightarrow \infty} F_{*}\left(x, D u_{k}(x)\right)=F_{*}(x, D u(x)) \text { a.e. } x \in B_{x_{0}}(r),
$$

where

$$
F_{*}(x, D u(x))=d_{F}\left(x_{0}, x\right)^{-\frac{n}{2}}\left[\frac{n-2}{2} J_{\alpha}\left(j_{\alpha} \frac{d_{F}\left(x_{0}, x\right)}{r}\right)-j_{\alpha} \frac{d_{F}\left(x_{0}, x\right)}{r} J_{\alpha}^{\prime}\left(j_{\alpha} \frac{d_{F}\left(x_{0}, x\right)}{r}\right)\right] .
$$

In (4.14) we used the chain rule, relation (2.9) and the inequality

$$
j_{\alpha} t \frac{J_{\alpha}^{\prime}\left(j_{\alpha} t\right)}{J_{\alpha}\left(j_{\alpha} t\right)} \leq \alpha \leq \frac{n-2}{2}, \quad t \in(0,1),
$$

which follows by relation (4.6). 
We now prove that the functions $x \mapsto F_{*}(x, D u(x))^{2}, x \mapsto \frac{u(x)^{2}}{d_{F}\left(x_{0}, x\right)^{2}}$ and $x \mapsto u(x)^{2}$ belong to $L^{1}\left(B_{x_{0}}(r)\right)$, respectively; these facts will be applied in the limiting process in (4.13) together with the Lebesgue dominated convergence theorem.

First, by Proposition 4.2/(i) (for $\beta=2$ ) and Proposition 4.1 it turns out that

$$
\begin{aligned}
\int_{B_{x_{0}}(r)} u(x)^{2} \mathrm{~d} V_{F}(x) & =\int_{B_{x_{0}}(r)} d_{F}\left(x_{0}, x\right)^{2-n} J_{\alpha}^{2}\left(j_{\alpha} \frac{d_{F}\left(x_{0}, x\right)}{r}\right) \mathrm{d} V_{F}(x) \\
& =r^{2-n} \int_{B_{x_{0}}(r)} h_{1}\left(\frac{d_{F}\left(x_{0}, x\right)}{r}\right) \mathrm{d} V_{F}(x) \\
& =\int_{0}^{r} \mathrm{~A}_{x_{0}}(\rho) \rho^{2-n} J_{\alpha}^{2}\left(j_{\alpha} \frac{\rho}{r}\right) \mathrm{d} \rho
\end{aligned}
$$

where $h_{1}$ comes from Proposition 4.2. Since $\rho \mapsto \frac{\operatorname{Vol}_{F}\left(B_{x_{0}}(\rho)\right)}{\rho^{n}}$ is non-increasing, it follows (see also (2.8)) that

$$
\operatorname{Vol}_{F}\left(B_{x_{0}}(\rho)\right) \leq \omega_{n} \rho^{n} \text { and } \mathrm{A}_{x_{0}}(\rho) \leq n \omega_{n} \rho^{n-1}, \quad \forall \rho>0
$$

Hence, by (3.9) and (4.15) we have

$$
\int_{0}^{r} \mathrm{~A}_{x_{0}}(\rho) \rho^{2-n} J_{\alpha}^{2}\left(j_{\alpha} \frac{\rho}{r}\right) \mathrm{d} \rho \leq n \omega_{n} \int_{0}^{r} \rho J_{\alpha}^{2}\left(j_{\alpha} \frac{\rho}{r}\right) \mathrm{d} \rho<+\infty .
$$

Second, again by Proposition 4.2/(i) (for $\beta=0$ ) and Proposition 4.1, one has

$$
\begin{aligned}
\int_{B_{x_{0}}(r)} \frac{u(x)^{2}}{d_{F}\left(x_{0}, x\right)^{2}} \mathrm{~d} V_{F}(x) & =\int_{B_{x_{0}}(r)} d_{F}\left(x_{0}, x\right)^{-n} J_{\alpha}^{2}\left(j_{\alpha} \frac{d_{F}\left(x_{0}, x\right)}{r}\right) \mathrm{d} V_{F}(x) \\
& =r^{-n} \int_{B_{x_{0}}(r)} h_{1}\left(\frac{d_{F}\left(x_{0}, x\right)}{r}\right) \mathrm{d} V_{F}(x) \\
& =\int_{0}^{r} \mathrm{~A}_{x_{0}}(\rho) \rho^{-n} J_{\alpha}^{2}\left(j_{\alpha} \frac{\rho}{r}\right) \mathrm{d} \rho \\
& \leq n \omega_{n} \int_{0}^{r} \rho^{-1} J_{\alpha}^{2}\left(j_{\alpha} \frac{\rho}{r}\right) \mathrm{d} \rho<\infty
\end{aligned}
$$

where we used (4.16) and the asymptotic property (3.9) together with the fact that $\alpha>0$.

By using the recurrence relation (4.3) and the eikonal equation (2.9), it turns out that

$F_{*}(x, D u(x))^{2}=d_{F}\left(x_{0}, x\right)^{-n}\left[\left(\frac{n-2}{2}-\alpha\right) J_{\alpha}\left(j_{\alpha} \frac{d_{F}\left(x_{0}, x\right)}{r}\right)+j_{\alpha} \frac{d_{F}\left(x_{0}, x\right)}{r} J_{\alpha+1}\left(j_{\alpha} \frac{d_{F}\left(x_{0}, x\right)}{r}\right)\right]^{2}$.

Therefore, in order to handle the term $\int_{B_{x_{0}}(r)} F_{*}(x, D u(x))^{2} \mathrm{~d} V_{F}(x)$, we have to study the behavior of three terms, coming from the development of (4.18). The first term appears precisely in (4.17). The second term is

$$
\begin{aligned}
0<I & :=\int_{B_{x_{0}}(r)} d_{F}\left(x_{0}, x\right)^{1-n} J_{\alpha}\left(j_{\alpha} \frac{d_{F}\left(x_{0}, x\right)}{r}\right) J_{\alpha+1}\left(j_{\alpha} \frac{d_{F}\left(x_{0}, x\right)}{r}\right) \mathrm{d} V_{F}(x) \\
& =r^{1-n} \int_{B_{x_{0}}(r)} h_{2}\left(\frac{d_{F}\left(x_{0}, x\right)}{r}\right) \mathrm{d} V_{F}(x) \\
& =\int_{0}^{r} \mathrm{~A}_{x_{0}}(\rho) \rho^{1-n} J_{\alpha}\left(j_{\alpha} \frac{\rho}{r}\right) J_{\alpha+1}\left(j_{\alpha} \frac{\rho}{r}\right) \mathrm{d} \rho \\
& \leq n \omega_{n} \int_{0}^{r} J_{\alpha}\left(j_{\alpha} \frac{\rho}{r}\right) J_{\alpha+1}\left(j_{\alpha} \frac{\rho}{r}\right) \mathrm{d} \rho<\infty
\end{aligned}
$$


where we used Proposition 4.2/(ii), Proposition 4.1 and relations (3.9) and (4.16). Finally, the third term is

$$
\begin{aligned}
J & :=\int_{B_{x_{0}}(r)} d_{F}\left(x_{0}, x\right)^{2-n} J_{\alpha+1}^{2}\left(j_{\alpha} \frac{d_{F}\left(x_{0}, x\right)}{r}\right) \mathrm{d} V_{F}(x) \\
& =r^{2-n} \int_{B_{x_{0}}(r)} h_{3}\left(\frac{d_{F}\left(x_{0}, x\right)}{r}\right) \mathrm{d} V_{F}(x)+\frac{r(n+2 \alpha)}{j_{\alpha}} I .
\end{aligned}
$$

Since $\rho \mapsto h_{3}(1)-h_{3}\left(\frac{\rho}{r}\right)$ is non-increasing on $(0, r]$, see Proposition $4.2 /$ (iii), which vanishes at $r$, we may apply Proposition 4.1 in order to obtain

$$
\begin{aligned}
\int_{B_{x_{0}}(r)} h_{3}\left(\frac{d_{F}\left(x_{0}, x\right)}{r}\right) \mathrm{d} V_{F}(x) & =-\int_{B_{x_{0}}(r)}\left[h_{3}(1)-h_{3}\left(\frac{d_{F}\left(x_{0}, x\right)}{r}\right)\right] \mathrm{d} V_{F}(x)+h_{3}(1) \operatorname{Vol}_{F}\left(B_{x_{0}}(r)\right) \\
& =-\int_{0}^{r} \mathrm{~A}_{x_{0}}(\rho)\left[h_{3}(1)-h_{3}\left(\frac{\rho}{r}\right)\right] \mathrm{d} \rho+h_{3}(1) \operatorname{Vol}_{F}\left(B_{x_{0}}(r)\right) \\
& =\int_{0}^{r} \mathrm{~A}_{x_{0}}(\rho) h_{3}\left(\frac{\rho}{r}\right) \mathrm{d} \rho
\end{aligned}
$$

Accordingly, by the latter relation, (4.19) and (4.16) one obtains

$$
\begin{aligned}
0<J & =r^{2-n} \int_{0}^{r} \mathrm{~A}_{x_{0}}(\rho) h_{3}\left(\frac{\rho}{r}\right) \mathrm{d} \rho+\frac{r(n+2 \alpha)}{j_{\alpha}} I \\
& =\int_{0}^{r} \mathrm{~A}_{x_{0}}(\rho) \rho^{2-n} J_{\alpha+1}^{2}\left(j_{\alpha} \frac{\rho}{r}\right) \mathrm{d} \rho \\
& \leq n \omega_{n} \int_{0}^{r} \rho J_{\alpha+1}^{2}\left(j_{\alpha} \frac{\rho}{r}\right) \mathrm{d} \rho<\infty .
\end{aligned}
$$

Now, we are in the position to apply the Lebesgue dominated convergence theorem in (4.13), obtaining

$$
\begin{aligned}
\int_{B_{x_{0}}(r)} F_{*}(x, D u(x))^{2} \mathrm{~d} V_{F}(x) \geq & {\left[\frac{(n-2)^{2}}{4}-\alpha^{2}\right] \int_{B_{x_{0}}(r)} \frac{u(x)^{2}}{d_{F}\left(x_{0}, x\right)^{2}} \mathrm{~d} V_{F}(x) } \\
& +S_{\alpha}\left(B_{x_{0}}(r)\right) \int_{B_{x_{0}}(r)} u(x)^{2} \mathrm{~d} V_{F}(x) .
\end{aligned}
$$

By (4.16) we have

$$
S_{\alpha}\left(B_{x_{0}}(r)\right)=j_{\alpha}^{2}\left(\frac{\omega_{n}}{\operatorname{Vol}_{F}\left(B_{x_{0}}(r)\right)}\right)^{\frac{2}{n}} \geq \frac{j_{\alpha}^{2}}{r^{2}} .
$$

Therefore, by using the integral representations from (4.15), (4.17), (4.19) and (4.20), the inequalities (4.18), (4.21) and (4.22) imply after some elementary rearrangement that

$$
\begin{gathered}
\int_{0}^{r} \mathrm{~A}_{x_{0}}(\rho) \rho^{2-n}\left\{J_{\alpha+1}^{2}\left(j_{\alpha} \frac{\rho}{r}\right)-2\left(\frac{n-2}{2}-\alpha\right) \frac{r}{j_{\alpha} \rho}\left[-J_{\alpha+1}\left(j_{\alpha} \frac{\rho}{r}\right)+\frac{\alpha r}{j_{\alpha} \rho} J_{\alpha}\left(j_{\alpha} \frac{\rho}{r}\right)\right] J_{\alpha}\left(j_{\alpha} \frac{\rho}{r}\right)-\right. \\
\left.-J_{\alpha}^{2}\left(j_{\alpha} \frac{\rho}{r}\right)\right\} \mathrm{d} \rho \geq 0 .
\end{gathered}
$$

We observe that for the second term in the above integrand we can apply the recurrence relation (4.3), obtaining

$$
\int_{0}^{r} \mathrm{~A}_{x_{0}}(\rho) \rho^{2-n}\left[J_{\alpha+1}^{2}\left(j_{\alpha} \frac{\rho}{r}\right)-2\left(\frac{n-2}{2}-\alpha\right) \frac{r}{j_{\alpha} \rho} J_{\alpha}^{\prime}\left(j_{\alpha} \frac{\rho}{r}\right) J_{\alpha}\left(j_{\alpha} \frac{\rho}{r}\right)-J_{\alpha}^{2}\left(j_{\alpha} \frac{\rho}{r}\right)\right] \mathrm{d} \rho \geq 0 .
$$


Now, we change the variable $\rho=r t, t \in(0,1)$, which implies

$$
\int_{0}^{1} \mathrm{~A}_{x_{0}}(r t) t^{2-n}\left[J_{\alpha+1}^{2}\left(j_{\alpha} t\right)-2\left(\frac{n-2}{2}-\alpha\right) \frac{1}{j_{\alpha} t} J_{\alpha}^{\prime}\left(j_{\alpha} t\right) J_{\alpha}\left(j_{\alpha} t\right)-J_{\alpha}^{2}\left(j_{\alpha} t\right)\right] \mathrm{d} t \geq 0 .
$$

Since the latter inequality is valid for every $r>0$, an integration with respect to $r$ yields

$$
\int_{0}^{1} \operatorname{Vol}_{F}\left(B_{x_{0}}(r t)\right) t^{1-n}\left[J_{\alpha+1}^{2}\left(j_{\alpha} t\right)-2\left(\frac{n-2}{2}-\alpha\right) \frac{1}{j_{\alpha} t} J_{\alpha}^{\prime}\left(j_{\alpha} t\right) J_{\alpha}\left(j_{\alpha} t\right)-J_{\alpha}^{2}\left(j_{\alpha} t\right)\right] \mathrm{d} t \geq 0 .
$$

Due to (2.7) and the latter inequality, the assumptions in Proposition 4.3 are fulfilled by the nonincreasing function $\rho \mapsto \frac{\operatorname{Vol}_{F}\left(B_{x_{0}}(\rho)\right)}{\rho^{n}}, \rho \in(0, \infty)$. Therefore, it yields that

$$
\operatorname{Vol}_{F}\left(B_{x_{0}}(\rho)\right)=\omega_{n} \rho^{n}, \quad \forall \rho>0 .
$$

We note that on $(M, F)$ with nonnegative $n$-Ricci curvature the latter relation does not depend on $x_{0}$, thus

$$
\operatorname{Vol}_{F}\left(B_{x}(\rho)\right)=\omega_{n} \rho^{n}, \quad \forall x \in M, \rho>0,
$$

which implies that the flag curvature on $(M, F)$ is identically zero.

Case II: $\alpha=0$ whenever $n=2$. The proof is similar to Case I. Let $r>0$ be arbitrarily fixed. Instead of the function from (4.12), we consider

$$
u(x):=J_{0}\left(j_{0} \frac{d_{F}\left(x_{0}, x\right)}{r}\right), x \in B_{x_{0}}(r) .
$$

After a similar approximation procedure as above, we obtain by (BPV) that

$$
\int_{B_{x_{0}}(r)} F_{*}(x, D u(x))^{2} \mathrm{~d} V_{F}(x) \geq S_{0}\left(B_{x_{0}}(r)\right) \int_{B_{x_{0}}(r)} u(x)^{2} \mathrm{~d} V_{F}(x) .
$$

Since

$$
F_{*}(x, D u(x))=-\frac{j_{0}}{r} J_{0}^{\prime}\left(j_{0} \frac{d_{F}\left(x_{0}, x\right)}{r}\right) F_{*}\left(x, D d_{F}\left(x_{0}, \cdot\right)(x)\right)=\frac{j_{0}}{r} J_{1}\left(j_{0} \frac{d_{F}\left(x_{0}, x\right)}{r}\right) \quad \text { a.e. } x \in B_{x_{0}}(r),
$$

and

the inequality (4.23) implies

$$
S_{0}\left(B_{x_{0}}(r)\right)=j_{0}^{2} \frac{\omega_{n}}{\operatorname{Vol}_{F}\left(B_{x_{0}}(r)\right)} \geq \frac{j_{0}^{2}}{r^{2}}
$$

$$
\int_{B_{x_{0}}(r)}\left[J_{1}^{2}\left(j_{0} \frac{d_{F}\left(x_{0}, x\right)}{r}\right)-J_{0}^{2}\left(j_{0} \frac{d_{F}\left(x_{0}, x\right)}{r}\right)\right] \mathrm{d} V_{F}(x) \geq 0 .
$$

By the latter inequality we obtain

$$
\int_{0}^{1} \operatorname{Vol}_{F}\left(B_{x_{0}}(r t)\right) t^{-1}\left[J_{1}^{2}\left(j_{0} t\right)-J_{0}^{2}\left(j_{0} t\right)\right] \mathrm{d} t \geq 0 .
$$

It remains to apply Proposition 4.3 to obtain $\operatorname{Vol}_{F}\left(B_{x_{0}}(\rho)\right)=\omega_{n} \rho^{n}, \rho>0$.

Proof of Theorem 1.3. "(i) $\Rightarrow$ (ii)" Since $(M, F)$ is Berwaldian, we have $\mathrm{Ric}_{n}=\mathrm{Ric}$, see Shen [23], and we can apply Theorem 1.2, obtaining that the flag curvature on $(M, F)$ is identically zero. Note that any Berwald space with vanishing flag curvature is necessarily a locally Minkowski space, see Bao, Chern and Shen [4, Sect. 10.5]. Now, the relation $\operatorname{Vol}_{F}\left(B_{x}(\rho)\right)=\omega_{n} \rho^{n}$ for all $x \in M$ and $\rho>0$ implies that $(M, F)$ is in fact isometric to a Minkowski space.

"(ii) $\Rightarrow(\mathrm{i}) "$ It follows directly by the first part of Theorem 1.1. 


\section{Application in PDEs: Proof of Theorem 1.4}

Proof of Theorem 1.4. Let us assume first that $\left(\mathcal{P}_{\alpha, \lambda}\right)$ has a nonzero solution, i.e., there exists $u \in W_{0}^{1,2}\left(B_{0}^{F}(1)\right)_{+} \backslash\{0\}$ such that

$$
-\Delta_{F} u(x)-\left[\frac{(n-2)^{2}}{4}-\alpha^{2}\right] \frac{u(x)}{F(x)^{2}}+\lambda u(x)=u(x)^{p-1}, \quad x \in B_{0}^{F}(1) .
$$

Due to Theorem 1.1, the function $u^{\star}(x)=F(x)^{\frac{2-n}{2}} J_{\alpha}\left(j_{\alpha} F(x)\right)$ belongs to $W_{0}^{1,2}\left(B_{0}^{F}(1)\right)$ and $u^{\star}(x)>0$ for every $x \in B_{0}^{F}(1)$. Moreover, the differential equation (3.7) or a direct calculation yields that

$$
-\Delta_{F} u^{\star}(x)-\left[\frac{(n-2)^{2}}{4}-\alpha^{2}\right] \frac{u^{\star}(x)}{F(x)^{2}}=S_{\alpha}\left(B_{0}^{F}(1)\right) u^{\star}(x), \quad x \in B_{0}^{F}(1) .
$$

Note that $S_{\alpha}\left(B_{0}^{F}(1)\right)=j_{\alpha}^{2}$. By multiplying the equation (5.1) by $u^{\star}$, an integration by parts and (5.2) give that

$$
\left(\lambda+j_{\alpha}^{2}\right) \int_{B_{0}^{F}(1)} u^{\star}(x) u(x) \mathrm{d} x=\int_{B_{0}^{F}(1)} u^{\star}(x) u(x)^{p-1} \mathrm{~d} x>0,
$$

which immediately yields $\lambda>-j_{\alpha}^{2}$.

Conversely, let us assume that $\lambda>-j_{\alpha}^{2}$ and define the positive number

$$
c_{\alpha, \lambda}:= \begin{cases}\min \left(1,1+\frac{\lambda}{j_{0}^{2}}\right), & \text { if } n=2(\text { and } \alpha=0), \\ \frac{4 \alpha^{2}}{(n-2)^{2}} \min \left(1,1+\frac{\lambda}{j_{\alpha}^{2}}\right), & \text { if } n \geq 3(\text { and } \alpha>0) .\end{cases}
$$

Due to the validity of (BPV) (see Theorem 1.1), we have for every $u \in W_{0}^{1,2}\left(B_{0}^{F}(1)\right)$ that

$$
\mathcal{K}_{\alpha, \lambda}^{2}(u):=\int_{B_{0}^{F}(1)}\left\{F_{*}(D u(x))^{2}-\left[\frac{(n-2)^{2}}{4}-\alpha^{2}\right] \frac{u(x)^{2}}{F(x)^{2}}+\lambda u(x)^{2}\right\} \mathrm{d} x \geq c_{\alpha, \lambda} \int_{B_{0}^{F}(1)} F_{*}(D u(x))^{2} \mathrm{~d} x .
$$

According to the classical Poincaré inequality and the latter inequality, $u \mapsto \mathcal{K}_{\alpha, \lambda}^{1 / 2}(u)$ defines a norm on $W_{0}^{1,2}\left(B_{0}^{F}(1)\right)$, equivalent to the usual one.

Let $g, G: \mathbb{R} \rightarrow[0, \infty)$ be defined by $g(s)=s_{+}^{p-1}$ and $G(s)=\frac{s_{+}^{p}}{p}$, where $s_{+}=\max (s, 0)$. We associate with problem $\left(\mathcal{P}_{\alpha, \lambda}\right)$ its energy functional $\mathcal{E}_{\alpha, \lambda}: W_{0}^{1,2}\left(B_{0}^{F}(1)\right) \rightarrow \mathbb{R}$ defined by

$$
\mathcal{E}_{\alpha, \lambda}(u)=\frac{1}{2} \mathcal{K}_{\alpha, \lambda}^{2}(u)-\int_{B_{0}^{F}(1)} G(u(x)) \mathrm{d} x .
$$

In a standard manner we can prove that $\mathcal{E}_{\alpha, \lambda} \in C^{1}\left(W_{0}^{1,2}\left(B_{0}^{F}(1)\right) ; \mathbb{R}\right)$. Moreover, since $W_{0}^{1,2}\left(B_{0}^{F}(1)\right)$ can be compactly embedded into $L^{p}\left(B_{0}^{F}(1)\right)\left(p \in\left(2,2^{*}\right)\right)$ and $s \mapsto g(s)$ verifies the usual AmbrosettiRabinowitz condition (see e.g. Willem [25, Lemma 1.20]), it turns out that $\mathcal{E}_{\alpha, \lambda}$ satisfies the PalaisSmale condition at each level. Moreover, since $p>2, \mathcal{E}_{\alpha, \lambda}$ satisfies also the mountain pass geometry; namely,

- there exists a sufficiently small $\rho>0$ such that

$$
\inf _{\mathcal{K}_{\alpha, \lambda}(u)=\rho} \mathcal{E}_{\alpha, \lambda}(u)>0=\mathcal{E}_{\alpha, \lambda}(0) ;
$$

- for sufficiently large $t>0$ and $u^{\star}$ coming from (5.2) one has

$$
\mathcal{E}_{\alpha, \lambda}\left(t u^{\star}\right)=t^{2} \mathcal{K}_{\alpha, \lambda}^{2}\left(u^{\star}\right)-t^{p} \int_{B_{0}^{F}(1)} G\left(u^{\star}(x)\right) \mathrm{d} x<0 .
$$


Therefore, by the mountain pass theorem it follows the existence of a critical point $u \in W_{0}^{1,2}\left(B_{0}^{F}(1)\right)$ for $\mathcal{E}_{\alpha, \lambda}$ with positive energy level (thus $u \neq 0$ ), which is precisely a weak solution to problem

$$
\left\{\begin{array}{l}
-\Delta_{F} u(x)-\left[\frac{(n-2)^{2}}{4}-\alpha^{2}\right] \frac{u(x)}{F(x)^{2}}+\lambda u(x)=(u(x))_{+}^{p-1}, \quad x \in B_{0}^{F}(1) ; \\
u \in W_{0}^{1,2}\left(B_{0}^{F}(1)\right) .
\end{array}\right.
$$

Multiplying the latter equation by $u_{-}(x)=\min (u(x), 0)$, an integration on $B_{0}^{F}(1)$ gives that $\mathcal{K}_{\alpha, \lambda}^{2}\left(u_{-}\right)=$ 0 , which implies $u_{-}=0$. Therefore, $u \geq 0$ is a solution for the original problem $\left(\mathcal{P}_{\alpha, \lambda}\right)$, which concludes the proof.

Acknowledgment. The authors thank Árpád Baricz for useful conversations on Bessel functions.

\section{REFERENCES}

[1] Adimurthi, N. Chaudhuri, M. Ramaswamy, An improved Hardy-Sobolev inequality and its applications, Proc. Amer. Math. Soc. 130 (2002), 489-505.

[2] A. Alvino, V. Ferone, P.-L. Lions, G. Trombetti, Convex symmetrization and applications, Ann. Inst. H. Poincaré Anal. Non Linéaire 14 (1997), no. 2, 275-293.

[3] G. Barbatis, S. Filippas, A. Tertikas, A unified approach to improved $L^{p}$ Hardy inequalities with best constants, Trans. Amer. Math. Soc. 356 (2004), 2169-2196.

[4] D. Bao, S. S. Chern, Z. Shen, Introduction to Riemann-Finsler Geometry, Graduate Texts in Mathematics, 200, Springer Verlag, 2000.

[5] E. Berchio, D. Ganguly, G. Grillo, Sharp Poincaré-Hardy and Poincaré-Rellich inequalities on the hyperbolic space, J. Funct. Anal. 272 (2017), no. 4, 1661-1703.

[6] H. Brezis, J. L. Vázquez, Blowup solutions of some nonlinear elliptic problems, Revista Mat. Univ. Complutense Madrid 10 (1997), 443-469.

[7] G. Carron, Inégalités de Hardy sur les variétés riemanniennes non-compactes, J. Math. Pures Appl. (9) 76 (1997), no. $10,883-891$.

[8] G. Carron, Inégalités isopérimétriques de Faber-Krahn et consequences, Publications de l'Institut Fourier, $220,1992$.

[9] S.-Y. Cheng, Eigenvalue comparison theorems and its geometric applications, Math. Z. 143 (1975), $289-297$.

[10] L. D'Ambrosio, S. Dipierro, Hardy inequalities on Riemannian manifolds and applications, Ann. Inst. H. Poincaré Anal. Non Linéaire, 31 (2014), no. 3, 449-475.

[11] A. Erdélyi, W. Magnus, F. Oberhettinger, F. Tricomi, Higher transcendental functions. Vols. I, II. Based, in part, on notes left by Harry Bateman. McGraw-Hill Book Company, Inc., New York-Toronto-London, 1953.

[12] L. Esposito, C. Trombetti, Convex symmetrization and Pólya-Szegö inequality, Nonlinear Anal. 56 (2004), no. 1, 43-62.

[13] C. Farkas, A. Kristály, C. Varga, Singular Poisson equations on Finsler-Hadamard manifolds, Calc. Var. Partial Differential Equations 54 (2015), no. 2, 1219-1241.

[14] N. Ghoussoub, A. Moradifam, Bessel pairs and optimal Hardy and Hardy-Rellich inequalities, Math. Ann. 349 (2011), no. 1, 1-57.

[15] N. Ghoussoub, A. Moradifam, On the best possible remaining term in the Hardy inequality, Proc. Natl. Acad. Sci. USA 105 (2008), no. 37, 13746-13751.

[16] I. Kombe, M. Özaydin, Improved Hardy and Rellich inequalities on Riemannian manifolds, Trans. Amer. Math. Soc. 361 (2009), no. 12, 6191-6203.

[17] I. Kombe, M. Özaydin, Hardy-Poincaré, Rellich and uncertainty principle inequalities on Riemannian manifolds, Trans. Amer. Math. Soc. 365 (2013), no. 10, 5035-5050.

[18] A. Kristály, Sharp uncertainty principles on Riemannian manifolds: the influence of curvature, J. Math. Pures Appl., in press. DOI: 10.1016/j.matpur.2017.09.002.

[19] A. Kristály, S. Ohta, Caffarelli-Kohn-Nirenberg inequality on metric measure spaces with applications, Math. Ann. 357 (2013), no. 2, 711-726.

[20] F. W. J. Olver, D. W. Lozier, R. F. Boisvert, C. W. Clark (eds.), NIST Handbook of Mathematical Functions, Cambridge University Press, Cambridge, 2010.

[21] S. Ohta, Finsler interpolation inequalities, Calc. Var. Partial Differential Equations 36 (2009), no. 2, 211-249.

[22] S. Ohta, K.-T. Sturm, Heat flow on Finsler manifolds. Comm. Pure and Appl. Math. 62 (2009), no. 10, $1386-1433$.

[23] Z. Shen, Volume comparison and its applications in Riemann-Finsler geometry, Adv. Math. 128 (1997), no. 2, 306-328. 
[24] J. Van Schaftingen, Anisotropic symmetrization, Ann. Inst. H. Poincaré Anal. Non Linéaire, 23 (2006), no. 4, 539-565.

[25] M. Willem, Minimax theorems. Progress in Nonlinear Differential Equations and their Applications, 24. Birkhäuser Boston, Inc., Boston, MA, 1996.

[26] Q. Yang, D. Su, Y. Kong, Hardy inequalities on Riemannian manifolds with negative curvature, Commun. Contemp. Math. 16(2) (2014), Article ID: 1350043, 24 pp.

Institute of Applied Mathematics, Óbuda University, Budapest, Hungary \& Department of Economics, Babeş-Bolyai University, Cluj-Napoca, Romania

E-mail address: kristaly.alexandru@nik.uni-obuda.hu; alexandrukristaly@yahoo.com

Institute of Applied Mathematics, Óbuda University, Budapest, Hungary

E-mail address: szakal@uni-obuda.hu 